\title{
Thermal Diffuse X-Ray-Scattering Studies of the Water-Vapor Interface
}

\section{Citation}

Schwartz, D. K., M. L. Schlossman, E. H. Kawamoto, G. J. Kellogg, Peter S. Pershan, and B. M. Ocko. 1990. Thermal diffuse $x$-ray-scattering studies of the water-vapor interface. Physical Review A 41(10): 5687-5690.

\section{Published Version}

doi:10.1103/PhysRevA.41.5687

\section{Permanent link}

http://nrs.harvard.edu/urn-3:HUL.InstRepos:8609120

\section{Terms of Use}

This article was downloaded from Harvard University's DASH repository, and is made available under the terms and conditions applicable to Other Posted Material, as set forth at http:// nrs.harvard.edu/urn-3:HUL.InstRepos:dash.current.terms-of-use\#LAA

\section{Share Your Story}

The Harvard community has made this article openly available.

Please share how this access benefits you. Submit a story.

Accessibility 


\title{
Thermal diffuse $x$-ray-scattering studies of the water-vapor interface
}

\author{
D. K. Schwartz, M. L. Schlossman, E. H. Kawamoto, G. J. Kellogg, and P. S. Pershan \\ Department of Physics and Division of Applied Sciences, Harvard University, Cambridge, Massachusetts 02138 \\ B. M. Ocko \\ Department of Physics, Brookhaven National Laboratory, Upton, New York 11973
}

(Received 8 December 1989)

\begin{abstract}
Agreement between the theoretical and measured resolution dependence of $\mathrm{x}$-ray specular reflection from the $\mathrm{H}_{2} \mathrm{O}$-vapor interface shows that the macroscopic capillary model for surface roughness can be extended to length scales as small as $400 \AA$. Agreement between measured thermal diffuse scattering data and the theoretical form, with no significant adjustable parameters, independently leads to similar conclusions.
\end{abstract}

\section{INTRODUCTION}

The statistical physics of liquid surfaces has been the subject of extensive theoretical studies, however, experiments capable of providing relevant data have only recently become available. ${ }^{1-8}$ One interesting development has been the interpretation of $\mathrm{x}$-ray reflectivity measurements in terms of a finite microscopic width, of the liquid-vapor interface, that is dominated by roughness due to thermally excited capillary waves. ${ }^{4-6}$ According to this interpretation, the specular reflectivity can be represented by $R\left(Q_{z}\right)=R_{F}\left(Q_{z}\right) \exp \left(-Q_{z}^{2} \sigma^{2}\right)$ where, $R_{F}\left(Q_{z}\right)$ is the Fresnel reflectivity for a smooth, sharp interface. For an incident angle $\alpha$ and wavelength $\lambda$, the wave-vector transfer $Q_{z}=(4 \pi / \lambda) \sin (\alpha)$. The interfacial width $\sigma$ has been represented as a combination of a local microscopic electron-density width $\sigma_{0}$ and the capillarywave contribution,

$$
\sigma^{2}=\sigma_{0}^{2}+\frac{k_{B} T}{4 \pi^{2} \gamma} \int_{A_{s}} \frac{1}{q^{2}+k_{g}^{2}} d \mathbf{q},
$$

where $k_{B} T$ is the Boltzman constant times the temperature, $\gamma$ is the macroscopic surface tension $k_{g}^{2}=\rho_{m} g / \gamma$, where $\rho_{m}$ is the mass density, and $A_{s}$ is a circular region with a rectangular cutout in reciprocal space. The inner boundary of $A_{s}$ is determined by the known resolution of the $\mathrm{x}$-ray reflectometer such that $\mathrm{x}$-rays scattered by capillary waves with wave vectors $q$ outside of the inner boundary, and thus contained in $A_{s}$, are deflected outside the angular acceptance of a detector centered on the position for specular reflection. ${ }^{9}$ This scattering reduces $R\left(Q_{z}\right) / R_{F}\left(Q_{z}\right)$ to less than unity. Since long wavelength capillary waves scatter at small angles, the $\sigma$ measured by a detector with finite resolution is not affected by waves at small q. Consequently $\sigma^{2}$ is specified in terms of only two adjustable parameters: the intrinsic width of the electron density profile $\sigma_{0}$ and the radius $q_{\max }$ of the outer perimeter of $A_{s}$.

Since the origin of both $\sigma_{0}$ and $q_{\max }$ are microscopic (on the scale of angstroms) their effects cannot be separat- ed by measurements that are only sensitive to in-plane length scales greater than $500 \AA$. Nevertheless, previous work had argued the plausibility of various values of $\sigma_{0}$ for particular choices of $q_{\max }$. For example, assuming $q_{\max } \approx\left(\pi / r_{\text {mol }}\right)$, where the molecular radius of $\mathrm{H}_{2} \mathrm{O}$ was taken to be $1.93 \AA$, Braslau et al. ${ }^{5,6}$ were able to fit the measurements at one resolution from the $\mathrm{H}_{2} \mathrm{O}$ surface and they obtained an intrinsic width of $\sigma_{0}=1.8 \pm 0.2 \AA$. Using higher-purity $\mathrm{H}_{2} \mathrm{O}$ Daillant et al. ${ }^{4}$ measured slightly smaller values for $\sigma$, and consequently obtained satisfactory fits using a physically equivalent theoretical form with $\sigma_{0} \approx 0$. Braslau et al. also measured the reflectivity from $\mathrm{CCl}_{4}$ and $\mathrm{CH}_{3} \mathrm{OH}$, having surface tensions, approximately, $\frac{1}{3}$ that of $\mathrm{H}_{2} \mathrm{O}$, and demonstrated that if $\sigma_{0}$ were allowed to vary, the reflectivities from the surface of all three liquids were consistent with known surface tensions and plausible values for $\sigma_{0}{ }^{10}$ They also measured the reflectivity of $\mathrm{CCl}_{4}$ using two different resolutions. Although the measured values of $\sigma$ roughly agreed with the capillary-wave prediction, the values of $\sigma_{0}$ were not equal.

In this paper we present specular reflectivity from the surface of clean $\mathrm{H}_{2} \mathrm{O}$ using three resolutions in the ratio of $6.25: 2.5: 1.0$. The data for all three resolutions can simultaneously be fit with $\sigma_{0}=0$ and one common value of $q_{\max }=(\pi / 1.4) \AA^{-1}$. In addition, we present diffusescattering measurements that agree with a theoretical form for capillary waves that has no significant adjustable parameters., 6,7 The combination of the resolution dependence of the specular reflection and the diffuse scattering demonstrates that capillary waves with wavelengths of the order of 400-80000 $\AA$ contribute to the width of the water-vapor interface as expected from the macroscopic theory of Eq. (1).

\section{EXPERIMENTAL}

A Langmuir trough, milled from a solid piece of Teflon, was filled with water from a Millipore Milli- $Q+$ system. ${ }^{11}$ The entire apparatus was sealed in an alumi- 
num box filled with high-purity nitrogen. The absolute surface tension, measured in situ by a Wilhelmy plate was $\gamma=72.5 \pm 0.4 \mathrm{dyn} / \mathrm{cm}$ at a temperature $T=20 \pm 0.5^{\circ} \mathrm{C}$. The precision of repeated measurements on a given water surface was $0.01 \mathrm{dyn} / \mathrm{cm}$. Surface purity was assured by requiring that changes in the surface tension accompany the sweep of a Teflon barrier across the surface were less than $0.1 \mathrm{dyn} / \mathrm{cm}$ for an area compression by a factor of 4 .

The experiments were performed on beam line $X 22 b$ at the National Synchrotron Light Source (NSLS) at Brookhaven National Laboratory using a liquid-surface reflectometer that has been described elsewhere. ${ }^{5,12,13}$ For specular reflection, the wavelength $\lambda=1.2569 \pm 0.0002 \AA$ and for diffuse scattering $\lambda=1.3965 \pm 0.0002 \AA$. The size of the beam was defined by a slit with dimensions $h_{i} \times w_{i}$ [(height) $\times($ width $)$, where $h_{i} \approx 0.2 \mathrm{~mm}$ and $w_{i} \approx 3.0-4.0 \mathrm{~mm}$, which was approximately $400 \mathrm{~mm}$ from the monochromator. The distance $L$ from the monochromator to the sample and from sample to detector was approximately $600 \mathrm{~mm}$. The measured angular spread was $\Delta \alpha \approx 6 \times 10^{-5} \mathrm{rad}$ in the vertical and $\Delta \Phi \approx 1 \times 10^{-3} \mathrm{rad}$ in the horizontal. The magnitude of $h_{i}$ was chosen so that its length projected on the sample, was smaller than the length of the sample (about $85 \mathrm{~mm}$ ). The detector slit could be placed so the detector accepted radiation at an angle $\beta$ from the horizontal and at an azimuthal angle $\phi$. The dimensions $h_{d}$ and $w_{d}$ of the detector slit were set larger than the incident slit dimensions so the reflected beam could pass into the detector.

Considering the illuminated area on the liquid surface to be a uniform source for the diffuse scattering, the full widths at half maximum of vertical and horizontal angular spread accepted by the detector are $\Delta \beta \approx h_{d} / L$ and $\Delta \phi=w_{d} / L .{ }^{14}$ The wave vector difference between the scattered and incident radiation, or the wave-vector transfer, is given by $Q=q_{\text {out }}-q_{\text {in }}$ :

$$
\begin{aligned}
& Q_{z}=(2 \pi / \lambda)[\sin (\alpha)+\sin (\beta)], \\
& Q_{x}=(2 \pi / \lambda) \sin (\phi / 2)[\cos (\beta)+\cos (\alpha)],
\end{aligned}
$$

and

$$
Q_{y}=(2 \pi / \lambda) \cos (\phi / 2)[\cos (\beta)-\cos (\alpha)] .
$$

The specular condition, $\alpha=\beta$ and $\phi=0$ is equivalent to $Q_{x}=Q_{y}=0$. The interior dimensions of $A_{s}, \Delta Q_{x}$ and $\Delta Q_{y}$ are calculated from $\Delta \alpha, \Delta \beta$, and $\Delta \phi$.

\section{RESULTS}

To test the predicted dependence of $\sigma^{2}$ on the interior dimensions of $A_{s}$ the angular dependence of specular reflectivity was measured with detector heights $h_{d}$ of 0.8 , 2.0, and $5.0 \mathrm{~mm}$. The three data sets are plotted in Fig. 1 as $\ln \left[R\left(Q_{z}\right) / R_{F}\left(Q_{z}\right)\right]$ versus $Q_{z}^{2}$. As predicted by Eq. (1), reflectivity for the smaller slits decreases faster. The three data sets were simultaneously fit to the capillarywave model, with $\sigma_{0}=0$, varying only $q_{\max }$. Since

$$
\Delta Q_{y} \approx(2 \pi / \lambda) \cos (\phi / 2) \sin (\beta) \Delta \beta,
$$

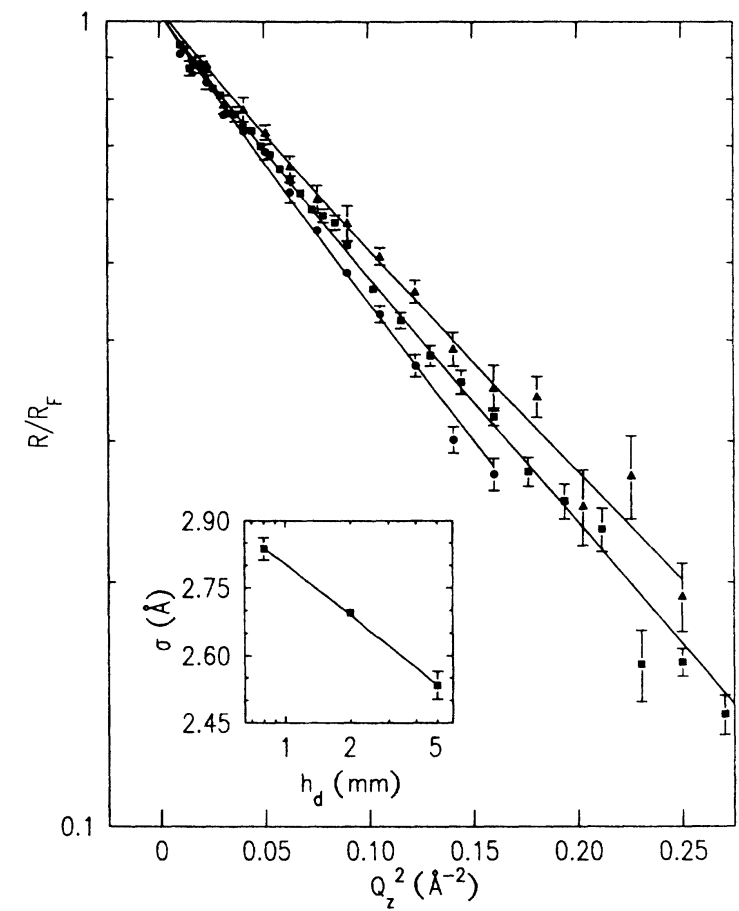

FIG. 1. Specular reflectivity data in the form of $R\left(Q_{z}\right) / R_{F}\left(Q_{z}\right)$ vs $Q_{z}^{2}$ for detector slit heights of $0.8 \mathrm{~mm}(O), 2.0$ $\mathrm{mm}(\mathbb{\square})$, and $5.0 \mathrm{~mm}(\Delta)$. The solid lines represent the best fit of the model discussed in the text. The inset shows the width of the surface profile plotted vs the detector slit height. The squares are derived from the fits to the data and the line is calculated from the theory at $Q_{z}=0.4 \AA^{-1}$.

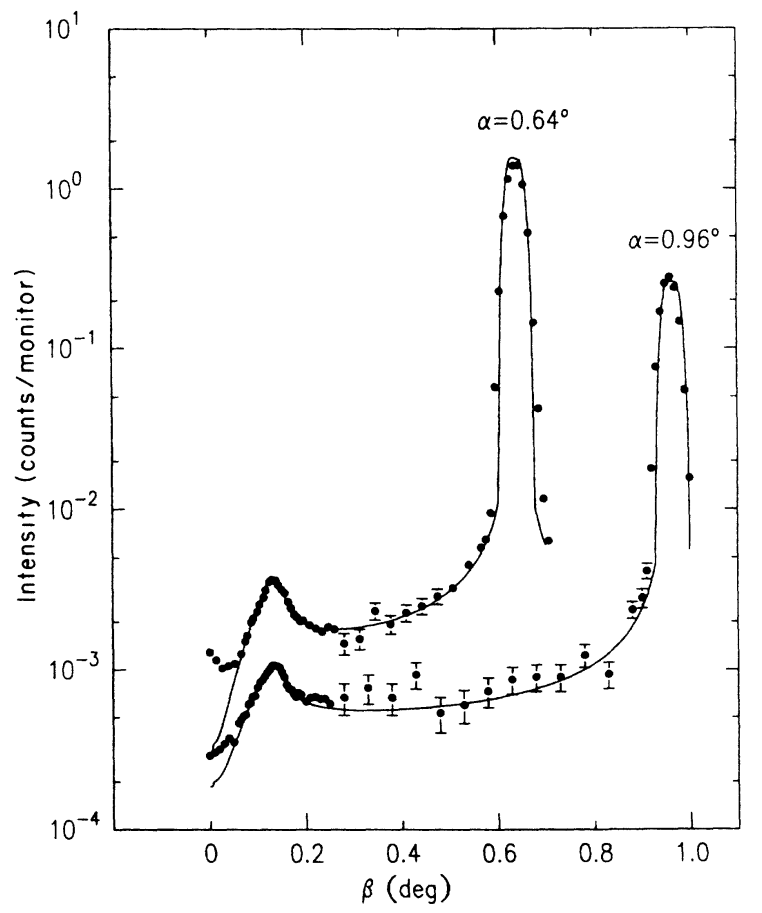

FIG. 2. Scattered intensity, in the plane of incidence, as a function of detector angle $\beta$ for incident angles $\alpha=0.64^{\circ}$ and $0.96^{\circ}$. The peaks at $\beta=\theta_{c} \approx 0.13^{\circ}$ are due to the surface scattering enhancement factor, the peaks at $\beta=\alpha$ are the specular reflectivity signals. The solid line is the theoretical prediction calculated with no significant adjustable parameters. 
both the interior boundary of $A_{s}$ and $\sigma^{2}$ vary with $Q_{z}$. The solid lines in Fig. 1 illustrating this fit correspond to $q_{\max }=(\pi / 1.4 \pm 0.1 \AA$ A $)$ and a $\chi^{2}=1.03$. The inset to Fig. 1 displays calculated values of $\left[\sigma^{2}\left(Q_{z}\right)\right]^{1 / 2}$ calculated from this fit for $Q_{z}=0.4 \AA^{-1}$.

In the specular position the reflectometer detects both the expected "specular signal," and diffuse scattering from windows, air, etc. Specular reflection is typically identified as the difference between scattering in the specular position and scattering at a small angle away from the specular position. This correctly subtracts away background that is approximately constant in angle. Since diffuse scattering from capillary waves varies as $1 /\left(q^{2}+k_{g}^{2}\right)$, and $k_{g}^{2}$ is much smaller than our resolution, this procedure also subtracts part of the scattering from capillary waves. Taking that into account, the solid lines in Fig. 1 correspond to the calculated difference between the specular and off-specular signals. ${ }^{15}$

According to the capillary-wave model, the intensity removed from the specular reflectivity by scattering from a capillary wave with wave vector $\mathbf{q}$ appears as diffuse scattering at finite $\mathbf{Q}=\left(q_{x}, q_{y}, Q_{z}\right)$. To examine this, $\mathrm{x}$ ray intensity was measured as a function of outgoing angles $\beta$, while keeping $\phi=0$ and the incident angle $\alpha$ fixed. Figure 2 illustrates data taken for $\alpha=0.64^{\circ}$ and $0.96^{\circ}$. The solid line is determined by the model, to be described below, which contains no significant adjustable parameters. The deviations at small $\beta$ correspond to small-angle scattering from the chamber window on the incident side of the trough. It is more prominent for smaller $\alpha$.

The theoretical prediction for the diffuse scattering $I_{D}$ is obtained by averaging the differential cross section $d \sigma / d \Omega=\left(r_{e} \rho\right)^{2}\left(A_{0} / \alpha\right) \Gamma f(\alpha) f(\beta)$ over the incident angular distribution and integrating over the angular acceptance of the detector. The cross sectional area of the incident beam is $A_{0}$ and the functions $f(\alpha)$ and $f(\beta)$ are the Fresnel surface scattering enhancement factors: $f(\beta) \approx\left(2 \beta / \theta_{c}\right)^{2} \sqrt{R_{F}(\beta)}$ (where the critical angle for total external reflection, $\left.\theta_{c}^{2} \equiv \rho e^{2} \lambda^{2} / \pi m c^{2}\right)$. They approach zero for $\alpha, \beta \rightarrow 0$, have a peak of amplitude 4 when $\alpha, \beta \approx \theta_{c}$, and level off to unity when $\beta \gg \theta_{c} .{ }^{6,16}$ Taking $\Gamma \equiv\left(k_{B} T / \gamma\right)\left(q^{2}+k_{g}^{2}\right)^{-1}$ :

$$
I_{D}=\left[I_{0} /\left(A_{0} \Delta \alpha\right)\right] \int_{\alpha-\Delta \alpha / 2}^{\alpha+\Delta \alpha / 2} d \alpha \int_{-\Delta \phi / 2}^{\Delta \phi / 2} d \phi \int_{\beta-\Delta \beta / 2}^{\beta+\Delta \beta / 2} d \beta d \sigma / d \Omega,
$$

where $\Delta \alpha, \Delta \beta$, and $\Delta \phi$ are the widths of the distributions of the respective angles and $I_{0}$ is the total incident flux. ${ }^{17}$ The displayed fits include a small constant background on the order of $10 \%$ of the intensity of the peak at $\beta=\theta_{c}$. In the small-angle approximation $Q_{x} \approx(2 \pi / \lambda) \phi$ and $Q_{y} \approx(\pi / \lambda)\left(\alpha^{2}-\beta^{2}\right)$ and the integral over $\phi$ can be done analytically. The solid lines in Fig. 2 are obtained by numerical integration of $I_{D}$ and by adding a $\delta$ function at $\alpha=\beta$, of amplitude $A_{\delta}=\exp \left(-Q_{z}^{2} \sigma^{2}\right)$, where

$$
\sigma^{2}=\frac{k_{B} T}{2 \pi \gamma} \int_{0}^{\infty} q d q /\left(q^{2}+k_{g}^{2}\right) .
$$

This term represents the true specular reflection, due to the gravitational cutoff of the $1 / q^{2}$ fluctuations, that would be observed with infinite resolution. ${ }^{18}$

\section{DISCUSSION}

The agreement between the resolution dependence of $\sigma^{2}$ and the specular reflectivity data out to $Q_{z} \approx 0.5 \AA^{-1}$ (i.e., $\beta \approx 0.05 \mathrm{rad}$ ) demonstrates that the macroscopic capillary-wave model is a good representation of the long-wavelength portion of the spectrum of surface excitations. In terms of the real-space fluctuations, this is equivalent to saying that the macroscopic description is a good representation of the height-height correlations in surface fluctuations over distances as short as the reciprocal of the coarsest resolution in $\Delta q_{y} \approx(2 \pi / \lambda)(\beta \Delta \beta / 2)$. For $Q_{z} \approx 0.5 \AA^{-1}, \quad \beta \approx 0.05 \mathrm{rad}$, and $\Delta Q_{z}=(2 \pi /$ $\lambda)(\Delta \beta / 2) \approx(2 \pi / 1.259 \AA)(5.0 \mathrm{~mm} / 600 \mathrm{~mm})$. This corresponds to the length $1 / \Delta q_{y}=1 /\left(\beta \Delta Q_{z}\right)=480 \AA$.
The agreement between theory and experiment - for both the amplitude and the line shape-for the diffuse scattering demonstrates that the macroscopic capillarywave model correctly represents the height-height correlation function over a region in reciprocal space that is bracketed by the transverse resolution $\Delta q_{x} \approx(2 \pi / \lambda)(\Delta \phi / 2)$ in one direction and $q_{y} \approx(2 \pi / \lambda)\left[\cos \left(\theta_{c}\right)-\cos (\theta)\right]$ in the other. These are typically $\Delta q_{x} \approx(2 \pi / 1.3965 \AA)[4 /(600 \times 2)]=0.015 \AA^{-1}$ and $q_{y} \approx(2 \pi / 1.3965 \AA)\left(1.4 \times 10^{-4}\right)=6.3 \times 10^{-4} \AA^{-1}$. The larger of these two corresponds to a characteristic length of $400 \AA$, in agreement with the length scale determined by specular reflection. The smallest wave vector for which diffuse scattering was measured is slightly larger than the spectrometer width $\Delta q_{y} \approx(2 \pi / 1.3965$ A) $\left[\cos \left(0.96^{\circ}\right)-\cos \left(0.9^{\circ}\right)\right]$ (e.g., Fig. 2) corresponding to approximately $8 \times 10^{4} \AA$.

Although it would be interesting to know if the capillary-wave model can be extended to even shorter lengths, or larger wave vectors, this would require either diffuse scattering, or specular reflectivity measurements at larger incident angles. Both of these measurements are limited by some combination of the maximum x-ray flux that can be applied to the sample and the diffuse background scattering from other sources. In principal, the higher flux and better collimation expected from future synchrotrons will probably enable these results to be extended to shorter lengths. In the meantime, one of the more important aspects of the present data is that it establishes confidence in the ability to use $\mathrm{x}$-rays to characterize the surface properties of simple liquids on the 
length scales of a few hundred angstroms to a few micrometers. In contrast, optical light scattering explores the surface on length scales of a micrometer to a millimeter. ${ }^{19}$

Measurements such as those reported here can now be applied to complex liquid surfaces with confidence that excess diffuse scattering above what is predicted from the capillary-wave model, or decreased reflectivity beyond what is expected from thermally excited capillary waves, can now be interpreted in terms of other microscopic models. For example, it is possible that there will be studies of excess diffuse scattering from compressed Langmuir monolayers on the surface of $\mathrm{H}_{2} \mathrm{O}$ that will yield information on the inhomogeneity of the monolayers in different parts of the two-dimensional phase dia- gram. There are numerous other possible systems where one might expect roughness beyond what is expected from capillary waves, and these can now all be studied with some confidence that the basic capillary wave effects can be understood.

\section{ACKNOWLEDGMENTS}

This work was supported by the National Science Foundation through grants to the Harvard Materials Research Laboratory, Grand Nos. NSF-DMR-88-12855 and NSF-DMR-86-14003. Research carried out at the NSLS, Brookhaven National Laboratory, is supported by the Department of Energy, Material Sciences, and Division of Chemical Sciences under Contract No. DEAC02-76CH00016.
${ }^{1}$ B. Widom, in Faraday Symp. R. Soc. Chem. 20, 16 (1981).

${ }^{2}$ Clive A. Croxton, Statistical Mechanics of Liquid Surfaces (Wiley, New York, 1980).

${ }^{3}$ S. A. Rice, Proc. Natl. Acad. Sci. 84, 4709 (1987).

4J. Daillant, L. Bosio, J. J. Benattar, and J. Meunier, Europhys. Lett. 8, 453 (1989).

${ }^{5}$ A. Braslau, M. Deutsch, P. S. Pershan, A. H. Weiss, J. AlsNielsen, and J. Bohr, Phys. Rev. Lett. 54, 114 (1985).

${ }^{6}$ A. Braslau, P. S. Pershan, G. Swislow, B. M. Ocko, and J. AlsNielsen, Phys. Rev. A 38, 2457 (1988).

${ }^{7}$ S. K. Sinha, E. B. Sirota, S. Garoff, and H. B. Stanley, Phys. Rev. B 38, 2297 (1988).

${ }^{8}$ D. Beaglehole, Phys. Rev. Lett. 58, 1434 (1987).

${ }^{9}$ The outer boundary of $A_{s}$ is defined by an upper cutoff similar to that of the Debye heat-capacity theory.

${ }^{10}$ See Ref. 6 above. The surface roughness for water in these measurements was artifically high due to surface contamination. The same may be true for the other liquids studied.

${ }^{11}$ Millipore Corp., Bedford, MA 01730.

12P. S. Pershan, J. Phys. (Paris) Colloq. 50, C7-1 (1989).

${ }^{13}$ J. Als-Nielsen and P. S. Pershan, Nucl. Instrum. Methods 208, 545 (1983).
${ }^{14}$ The full width at half maximum (FWHM) of angular distribution for a uniformly illuminated two slit configuration is determined by the larger of the two slits.

${ }^{15}$ The problem is actually even subtler, since were it not for the long-wavelength cutoff due to gravity, the capillary-wave spectrum would have a $1 / q^{2}$ singularity and from a strict point of view there would not be any specular reflection. See Refs. 6 and 7, also Y. Imbry and L. Gunther, Phys. Rev. B 3, 3939 (1971) and Y. Imbry, Crit. Rev. Solid State Mat. Sci. 8, 157 (1978).

${ }^{16}$ R. S. Becker, J. A. Golovchenko, and J. R. Patel, Phys. Rev. Lett. 50, 153 (1986).

${ }^{17}$ We have chosen a constant, or square wave, angular intensity distribution. This is a good approximation in this case, however, the results are almost identical for a trapezoidal distribution suggesting that they are insensitive to the exact form of the distribution.

${ }^{18}$ In the small- $Q_{z}$ limit this expression for $A_{\delta}$ corresponds to the sum rule $I_{0} R_{F} A_{\delta}+\int_{\text {all space }} I_{D}=I_{0} R_{F}$.

${ }^{19}$ R. Loudon, in Surface Excitations, edited by V. M. Agranovich and R. Loudon (Elsevier, New York, 1984), pp. 589-638. 